\title{
Aprendiendo el cálculo diferencial e integral con GeoGebra
}

\section{Learning Differential and Integral Calculus with GeoGebra}

\author{
Nilo Teodorico Colquepisco Paucar ${ }^{1,2 *}$ \\ Universidad Nacional de Cañete ${ }^{1}$ \\ Escuela Profesional de Ingeniería de Sistemas ${ }^{2}$ \\ *ncolquepisco@undc.edu.pe
}

DOI: https://doi.org/10.26871/killkana_tecnica.v3i2.484

\begin{abstract}
Resumen
En este artículo, se explica la mejora del aprendizaje de las derivadas e integrales mediante el uso del programa informático, GeoGebra, en estudiantes universitarios. En la enseñanza superior en el área de Matemáticas en la Escuela de Ingeniería de Sistemas. Una de las problemáticas que se presenta en la situación local y nacional es el insuficiente beneficio en las matemáticas superiores en los estudiantes. Las probables causalidades pueden ser la restricción del uso de los recursos didácticos, el uso exorbitante de la pizarra, limitados recursos bibliográficos a capacidad, una metodología tradicional donde los estudiantes son un ente pasivo y receptivos de los temas, y la no inserción de las novedades tecnológicas en el aula de clase. Por ello es importante el programa GeoGebra que facilita el aprendizaje, con el incentivo de resolver más ejercicios y con mucha motivación aprenden el cálculo diferencial e integral. Este trabajo tiene aplicación en temas de derivadas e integrales, las mismas que son mejoradas año tras año y evidencia la trascendencia de tal aplicación. El estudio se enmarcó dentro del enfoque cuantitativo, método hipotético deductivo, tipo aplicada, de diseño experimental- cuasi experimental, técnica la evaluación, así mismo la muestra seleccionada para realizar esta investigación la conformaron 60 alumnos en cada grupo del II ciclo de la carrera de Ingeniería de Sistemas.
\end{abstract}

Palabras clave: Aprendizaje, derivadas, GeoGebra, integrales.

\begin{abstract}
This article presents an explanation about the improvement of derivative and integral learning through the use of GeoGebra computer program, by university students attending the Systems Engineering school, in the Mathematics area. One of the problems faced in the local and national contexts is the students' lack of higher mathematics mastery. The possible causes could be the use restriction of didactic resources, the excessive use of the blackboard, limited bibliographic resources, the use of traditional methodologies where the students are passive and receptive entities of knowledge, and the lack of new technology implementation in the classroom. It is important to use the GeoGebra program because it facilitates learning on differential and integral calculus, while motivates the student to solve more exercises. This program can also be applied to derivatives and integrals learning, which improves each year evidencing the importance of the application. The study followed the quantitative approach, the method used was hypothetical deductive, with experimental-quasiexperimental design, the sample selected to carry out this research was formed by 60 students per group, attending the II cycle of Systems Engineering.
\end{abstract}

Key words: Learning, derivatives, GeoGebra, integrals.

\section{INTRODUCCIÓN}

La preocupación metodológica en la enseñanza - aprendizaje es una dificultad global, pues abarca a todo el planeta. La investigación está enfocada en el aprendizaje de las derivadas e integrales con la utilización del programa informático libre llamado GeoGebra, en alumnos de pregrado de la Universidad Nacional de Cañete.

En la actualidad, los profesores tienen como principal preocupación la enseñanza de las matemáticas con algún tipo de programa informático libre. En esta asignatura algunos procedimientos resultan tediosos y en otras incomprensibles, para ello se ha visto que la aplicación de un software, cambia el entorno del estudiante a un ambiente nuevo de participación, donde se descubren hechos con mucha facilidad y rapidez. Esta metodología provoca que el conocimiento sea significativo y que a la vez sea el punto de partida para generar aplicaciones directas al campo de estudio.

La Universidad Nacional de Cañete, no está ajena a tener como misión la creación beneficiosa para la población. Uno de los problemas que se encuentran en el ámbito universitario es la escasez del rendimiento en las matemáticas superiores en los estudiantes. La situación antes mencionada tiene como probables razones: el restringido 
uso de los medios educativos, la utilización exorbitante del pizarrón, el escaso uso de medios bibliográficos a su capacidad, que deriva en la creencia tradicional que los estudiantes son un ser abúlico y receptivos de contenidos. La no inserción de las nuevas tecnologías en las aulas universitarias, es una de las posibles razones que producirían como resultado el rendimiento muy bajo de las matemáticas en los estudiantes.

La formación actual de las matemáticas propone una formación experimental, en el progreso de la percepción del alumno para comprender la peculiaridad de las ideas que observan, y sostener una percepción habitual del problema constituye el propósito primordial de esa instrucción.

La metodología de adiestramiento de las matemáticas es profundamente dificultosa, y al transcurrir el tiempo el ser humano ha progresado una variedad de métodos para alcanzar la eficiencia de dicho procedimiento. Con el arribo de las novedades técnicas, en peculiar de los ordenadores, se abre un reciente campo de las pesquisas en cuanto a nuevos entornos de enseñanza y procedimiento, que permiten aprovechar la grandeza potencial de estos recursos.

Conde [1] manifiesta "la idoneidad del uso del software GeoGebra para la mejora del proceso de enseñanza - aprendizaje de las matemáticas en $4^{\circ}$ de E.S.O." La competencia adquirida con el uso de programa GeoGebra es adecuada en la enseñanza de las matemáticas. El autor también planteo como objetivo general evaluar la idoneidad del uso del software GeoGebra como herramienta didáctica en el proceso de enseñanza - aprendizaje de las matemáticas en el último curso de la educación secundaria obligatoria. El autor manifiesta que la aplicación del programa educativo GeoGebra dentro del salón es altamente favorable para la adquisición de competencias matemáticas así como para perfeccionar actitudes del estudiante.

Y así mismo Bello [2] desarrolló una investigación titulada: Mediación del software GeoGebra en el aprendizaje de programación lineal en alumnos del quinto grado de educación secundaria, donde orientó como propósito general trazar una propuesta de asignación mediadas por el pro- grama educativo GeoGebra que facilite el aprendizaje de la programación lineal y que facilite a los estudiantes transitar entre los Registros de Representación verbal, algebraico y gráfico al resolver problemas contextualizados en alumnos de quinto grado de E.S. de la I.E. La metodología empleada, en cuanto al tipo de investigación fue cualitativa. Se aplicó la técnica de entrevista y como instrumento ficha de actividades. Él nos manifiesta que estar familiarizados con el uso de un vocabulario nuevo especializado en geometría dinámica con Geogebra, puede obtener gráficos completos y no distorsionados al representar inecuaciones.

\section{El SOFTWARE GeoGebra}

Para López [3] el programa es un software matemático interactivo libre que está lleno de funcionalidades tendientes a simplificar las construcciones geométricas y algebraicas. Es un recurso educativo que se utiliza como herramienta didáctica en la enseñanza de la matemática. El programa educativo GeoGebra es de sencilla utilización a pesar de su complejidad.

La preparación es muy instintiva con clases especiales de manipulación del programa educativo GeoGebra, ni configuración de notas sofisticadas, tiene tres partes esenciales como son: barra de herramientas, ventana algebraica, ventana grafica y campo de entrada. "La presentación de la pantalla del programa cuenta con dos ventanas activas: una zona de dibujo en la que se crean y manipulan objetos geométricos: puntos; segmentos, rectas, vectores, triángulos, polígonos, círculos, arcos, cónicas, los mismos que en Cabri Geometre II; y otra donde aparecen las coordenadas de los puntos y las ecuaciones de las rectas y curvas trazadas que se actualizan simultáneamente con los cambios en la región gráfica" [3].

Las ventajas sobre otros software como Cabri Geometre II es que se pueden ingresar ecuaciones y coordenadas directamente, en la cual se permite manipular las variables relacionadas a números y vectores; además facilita evaluar las derivadas e integrales de funciones con una facilidad y dinamización.

TABLA I: Contraste del programa en tema de álgebra y cálculo

\begin{tabular}{|l|c|c|c|c|c|c|c|}
\hline Parámetro & Matlab & Derive & Derivator & Mathtype & Maxima & Octave & EulerMath \\
\hline Licencia gratis & & & $\mathrm{X}$ & & $\mathrm{X}$ & $\mathrm{X}$ & $\mathrm{X}$ \\
\hline $\begin{array}{l}\text { Soporta operaciones algebraicas } \\
\text { complejas }\end{array}$ & $\mathrm{X}$ & $\mathrm{X}$ & & & $\mathrm{X}$ & $\mathrm{X}$ & $\mathrm{X}$ \\
\hline Solución de matrices & $\mathrm{X}$ & $\mathrm{X}$ & & & $\mathrm{X}$ & $\mathrm{X}$ & $\mathrm{X}$ \\
\hline Solución de derivadas & $\mathrm{X}$ & $\mathrm{X}$ & $\mathrm{X}$ & & $\mathrm{X}$ & $\mathrm{X}$ & $\mathrm{X}$ \\
\hline Solución de integrales & $\mathrm{X}$ & $\mathrm{X}$ & & & $\mathrm{X}$ & $\mathrm{X}$ & $\mathrm{X}$ \\
\hline Permite gráficos en 3D & $\mathrm{X}$ & $\mathrm{X}$ & & & $\mathrm{X}$ & & $\mathrm{X}$ \\
\hline Permite gráficos en 2D & $\mathrm{X}$ & $\mathrm{X}$ & & & $\mathrm{X}$ & $\mathrm{X}$ & $\mathrm{X}$ \\
\hline Resultados confiables & $\mathrm{X}$ & $\mathrm{X}$ & & & $\mathrm{X}$ & $\mathrm{X}$ & $\mathrm{X}$ \\
\hline Variedad de herramientas & $\mathrm{X}$ & $\mathrm{X}$ & & & $\mathrm{X}$ & & \\
\hline Interface atractiva & $\mathrm{X}$ & $\mathrm{X}$ & & & & & \\
\hline
\end{tabular}


TABLA I: Contraste del programa en tema de álgebra y cálculo...(continuación)

\begin{tabular}{|l|c|c|c|c|c|c|c|}
\hline Parámetro & Matlab & Derive & Derivator & Mathtype & Maxima & Octave & EulerMath \\
\hline Fácil utilización & & $\mathrm{X}$ & & $\mathrm{X}$ & & & \\
\hline Variedad de idiomas & & & & $\mathrm{X}$ & & & \\
\hline Variedad de sistemas operativos & $\mathrm{X}$ & & & $\mathrm{X}$ & & & \\
\hline $\begin{array}{l}\text { Explotar resultados a otros forma- } \\
\text { tos }\end{array}$ & & $\mathrm{X}$ & & $\mathrm{X}$ & $\mathrm{X}$ & & \\
\hline
\end{tabular}

Archivo Edita Vista Opciones Herramientas Ventana Ayuda

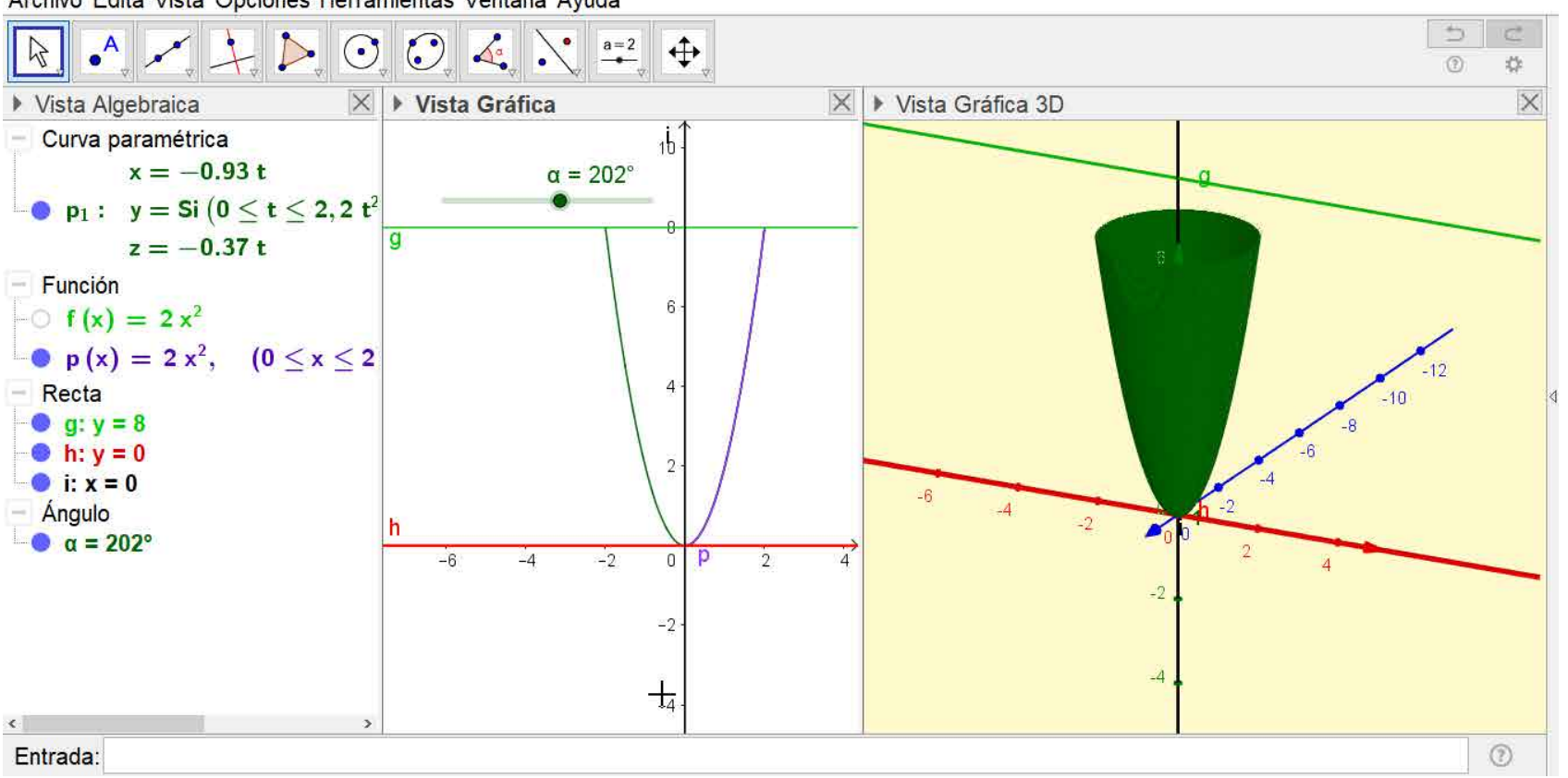

Fig. 1: Estructura del programa GeoGebra

\section{A. Principales funcionalidades del software GeoGebra}

LarRepresentación de funciones y sus respectivas gráficas, permite obtener los máximos y mínimos de la función.

Permite obtener la función derivada en cualquier orden con sus respectivas gráficas.

Se pueden integrar las funciones, es decir, obtener el área baja de la curva, y obtener el volumen de revolución de las funciones acotada.

El software permite una sencilla manipulación, y un trabajo favorable y beneficioso.

Puede ampliar la utilidad visual de tal manera, que permite tener otra visión de las matemáticas.

Presenta una variedad y cantidad de funcionalidades, como gráfica de funciones, trazados, áreas, etc.

Otra funcionalidades son los deslizadores, elementos con una gran capacidad, ya que permiten observar animaciones con facilidad. Ya sea la rotación de un triángulo, traslación de puntos, homotecia de una figura u otros.

\section{B. El Aprendizaje en el área de las Matemáticas}

Las Matemáticas como adquisición de habilidades intelectuales, mejora las tácticas cognoscitivas, y la habilidad motora o actitudes.

El conductismo como teoría, se apoya en los estudios del aprendizaje mediado condicionamiento y halla insignificante el estudio de procesos memorísticos superiores para la asimilación de la conducta del ser humano.

Barrón (1993) indica: "el aprendizaje por descubrimiento es un tipo de aprendizaje en el que el sujeto, en vez de recibir los contenidos de forma pasiva, descubre los conceptos y sus relaciones y los reordena para adaptarlos a su esquema cognitivo".

\section{Método inductivo}

Es el estudio de las pruebas que permite medir la probabilidad de los argumentos y un modo de razonar que lleva de lo particular a lo general.

\section{Método deductivo}

Es un modelo reflexivo que la conclusión se halla en forma implícita y conduce de lo general a lo particular. 
La inducción y la deducción no son formas diferentes de razonamiento, ambas son formas de inferencia.

Este método hace uso de la deducción por una conclusión sobre una premisa particular.

\section{Metodología}

El Perú es un país en vías del crecimiento, esto implica la necesidad de que los estudiantes en cualquier grado educativo tengan dentro de sus proyectos de vida, impulsar el desarrollo científico. Por tanto, involucrar la tecnología en los procesos educativos es una tarea importante en función de esa revolución, que de un giro definitivo en el aprendizaje de los estudiantes.

La investigación se enmarca al diseño cuasi experimental. Se determina el efecto que tendrá la utilización del programa educativo GeoGebra sobre el aprendizaje de las derivadas e integrales.

TABLA II: Diseño de la investigación

\begin{tabular}{lccc}
\hline Grupos & Prueba de la Investigación & Tratamiento & Prueba de salida \\
\hline GC & $\mathrm{O}_{1}$ & & $\mathrm{O}_{2}$ \\
GE & $\mathrm{O}_{3}$ & $\mathrm{X}$ & $\mathrm{O}_{4}$ \\
\hline
\end{tabular}

Fuente: Metodología de investigación Hernández, Fernández y Baptista, 2012

Donde:

GE y GC: son grupos experimental y control

$\mathbf{O}_{1}, \mathbf{O}_{3}$ : La prueba de entrada antes del tratamiento

$\mathbf{O}_{2}, \mathbf{O}_{4}$ : La prueba de salida posterior del tratamiento

X: Tratamiento con el programa educativo GeoGebra

TABLA III: Grupo control y experimental

\begin{tabular}{lcc}
\hline Grupo de Investigción & II ciclo de Ing. Sistemas & No. de alumnos \\
\hline Grupo de control & Turno de mañana & 50 \\
Grupo experimental & Turno tarde & 50 \\
\hline
\end{tabular}

Fuente: Registro de la UNDC

TABLA IV: Operacionalización del aprendizaje de las derivadas

\begin{tabular}{|c|c|c|c|c|}
\hline Dimensiones & Indicadores & Items & $\begin{array}{l}\text { Escala y } \\
\text { valores }\end{array}$ & Niveles y rangos \\
\hline \multirow{2}{*}{$\begin{array}{l}\text { Primeras derivadas de } \\
\text { funciones }\end{array}$} & Regla de derivación & $\mathrm{i} 1, \mathrm{i} 2, \mathrm{i} 3$ & \multirow{6}{*}{$\begin{array}{l}\text { Correcto (1) } \\
\text { incorrecto (0) }\end{array}$} & \multirow{6}{*}{$\begin{array}{l}\text { Excelente[19-20] } \\
\text { Muy Bueno[16-18] } \\
\text { Bueno[14-15] } \\
\text { Aprobado[11-13] } \\
\text { Desaprobado[0-10] }\end{array}$} \\
\hline & Resuelve ejercicios & $\mathrm{i} 4, \mathrm{i} 5, \mathrm{i} 6, \mathrm{i} 7$ & & \\
\hline $\begin{array}{l}\text { Interpretación } \\
\text { geométrica de la }\end{array}$ & Gráfica funciones & i8, i9, i10 & & \\
\hline derivada & Analiza la gráfica & $\mathrm{i} 11, \mathrm{i} 12, \mathrm{i} 13, \mathrm{i} 14$ & & \\
\hline \multirow{2}{*}{$\begin{array}{l}\text { Aplicación de las } \\
\text { derivadas }\end{array}$} & $\begin{array}{l}\text { Halla los máximos } \\
\text { y mínimos }\end{array}$ & $\mathrm{i} 15, \mathrm{i} 16, \mathrm{i} 17$ & & \\
\hline & $\begin{array}{l}\text { Interpreta la } \\
\text { aplicación }\end{array}$ & $\mathrm{i} 18, \mathrm{i} 19, \mathrm{i} 20$ & & \\
\hline
\end{tabular}

Fuente: Elaborado para el estudio 
TABLA V: Operacionalización del aprendizaje de las integrales

\begin{tabular}{|c|c|c|c|c|}
\hline Dimensiones & Indicadores & Items & $\begin{array}{l}\text { Escala y } \\
\text { valores }\end{array}$ & Niveles y rangos \\
\hline \multirow{2}{*}{$\begin{array}{l}\text { Cálculo de las } \\
\text { integrales indefinidas }\end{array}$} & $\begin{array}{l}\text { Regla de } \\
\text { integración }\end{array}$ & i1, i2, i3 & \multirow{6}{*}{ Correcto (1) } & \multirow{6}{*}{$\begin{array}{l}\text { Excelente[19-20] } \\
\text { Muy Bueno[16-18] } \\
\text { Bueno[14-15] } \\
\text { Aprobado[11-13] } \\
\text { Desaprobado[0-10] }\end{array}$} \\
\hline & Resuelve ejercicios & $\mathrm{i} 4, \mathrm{i} 5, \mathrm{i} 6, \mathrm{i} 7$ & & \\
\hline \multirow[t]{2}{*}{ Integrales de Riemann } & $\begin{array}{l}\text { Representa las } \\
\text { integrales de } \\
\text { Riemann }\end{array}$ & i8, i9, i10 & & \\
\hline & Resuelve ejercicios & $\mathrm{i} 11, \mathrm{i} 12, \mathrm{i} 13, \mathrm{i} 14$ & & \\
\hline \multirow{2}{*}{$\begin{array}{l}\text { Cálculo de los sólidos } \\
\text { de revolución }\end{array}$} & $\begin{array}{l}\text { Calcula áreas de } \\
\text { regiones planas }\end{array}$ & $\mathrm{i} 15, \mathrm{i} 16, \mathrm{i} 17$ & & \\
\hline & $\begin{array}{l}\text { Calcula volumen } \\
\text { de solidos }\end{array}$ & i18, i19, i20 & & \\
\hline
\end{tabular}

Fuente: Elaborado para el estudio

TABLA VI: Población y muestra

\begin{tabular}{lccc}
\hline Ciclo & Turno & No de Alumnos & $\%$ \\
\hline II & Mañana & 50 & 50 \\
II & Tarde & 50 & 50 \\
Total de alumnos & & 100 & 100 \\
\hline
\end{tabular}

Fuente: Registro de estudiantes

\section{RESUltados}

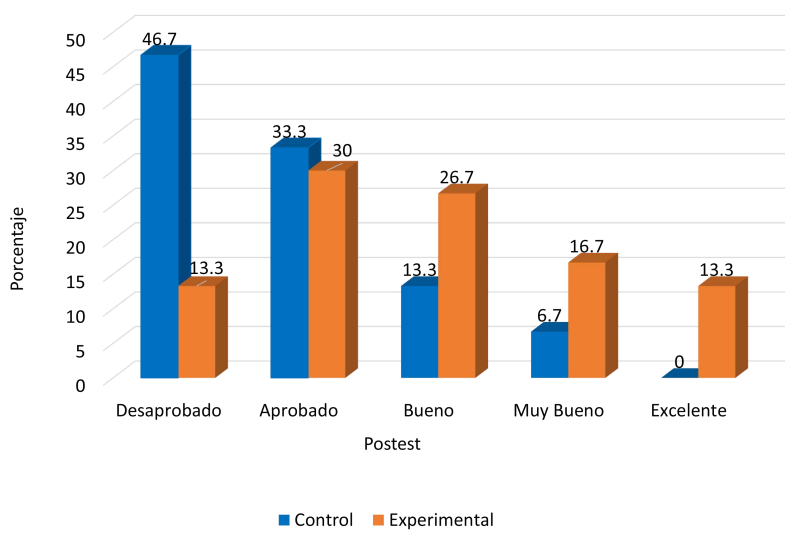

Fig. 2

Como se puede observar, los resultados tanto del grupo control como del grupo experimental, son muy diferentes. En el grupo de control 14 alumnos $(46.7 \%)$ se encuentran en el nivel desaprobado, 10 alumnos $(33.3 \%)$ se encuentran en el nivel aprobado, 4 alumnos $(13.3 \%)$ se encuentran en el nivel bueno, 2 alumnos $(6.7 \%)$ se encuentran en el nivel Muy bueno y 0 alumnos $(0 \%)$ se encuentran en el nivel excelente. Mientras que en el grupo experimental, existen 4 alumnos $(13.3 \%)$ que se encuentran en el nivel de desaprobado, 9 alumnos (30\%) se encuentra en el nivel de aprobado, 8 alumnos (26.7\%) se encuentran en el nivel de bueno, 5 alumnos (16.7\%) se encuentran en el nivel de muy bueno y 4 alumnos $(13.3 \%)$ se encuentran en el nivel de Excelente. Después de la aplicación del software Geogebra en el grupo experimental en el Postest, se observa que tanto el grupo de control como el grupo experimental son muy diferentes.

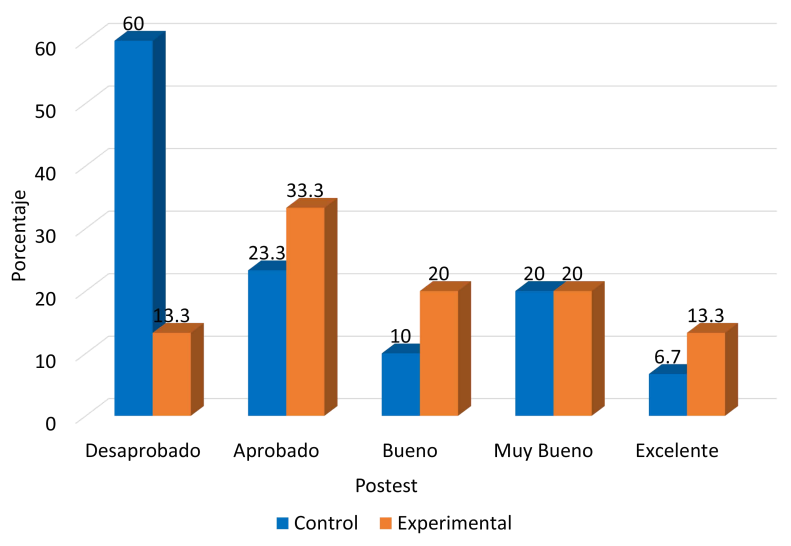

Fig. 3 
En el grupo de control, 18 alumnos $(60 \%)$ se encuentran en el nivel desaprobado, 7 alumnos $(23.3 \%)$ se encuentran en el nivel aprobado, 3 alumnos (10\%) se encuentran en el nivel bueno, 6 alumnos $(20 \%)$ se encuentran en el nivel Muy bueno y 2 alumnos $(6.7 \%)$ se encuentran en el nivel excelente. Mientras que en el grupo experimental existen 4 alumnos (13.3\%) que se encuentran en el nivel de desaprobado, 10 alumnos $(33.3 \%)$ se encuentra en el nivel de aprobado, 6 alumnos (20\%) se encuentran en el nivel de bueno, 6 alumnos (20\%) se encuentran en el nivel de muy bueno y 4 alumnos $(13.3 \%)$ se encuentran en el nivel de Excelente.

\section{CONCLUSIONES Y RECOMENDACIONES}

Luego de haber estudiado cuidadosamente el programa educativo GeoGebra para el ámbito de la matemática y la ingeniería, se concluye que se evidencia que existe una diversidad de aplicaciones matemáticas que pueden facilitar la labor de los profesores y los alumnos antes de la educación.

Se debe considerar la utilización de los programas para fines pedagógicos y no se vuelvan una necesidad para resolver una operación matemática.

Con estos resultados se ha verificado que la aplicación del programa GeoGebra influye en el aprendizaje de las derivadas $(\mathrm{Z}=-3,500$ y $\mathrm{Sig} .=0,000)$ y el aprendizaje de las integrales $(Z=-4,162$ y Sig. $=0,000)$ en los estudiantes del II ciclo de ingeniería de Sistemas de la Universidad Nacional de Cañete.

Promover que la Universidad Nacional de Cañete incluya en capacitaciones de la preparación de las matemáticas el programa educativo GeoGebra, para que favorezca el proceso de enseñanza de las derivadas e integrales en los estudiantes del II ciclo de la escuela profesional de ingeniería de Sistemas de la Universidad Nacional de Cañete.

\section{REFERENCIAS}

[1] R. Conde-Serrano, "Idoneidad del uso del software geogebra para la mejora del proceso enseñanzaaprendizaje de las Matemáticas en $4^{\circ}$ de ESO," 2013.

[2] J. B. Bello Durand, "Mediación del software Geogebra en el aprendizaje de programación lineal en alumnos del quinto grado de educación secundaria.," 2013.

[3] N. Ruiz López, "Análisis del desarrollo de competencias geométricas y didácticas mediante el software de geometría dinámica GeoGebra en la formación inicial del profesorado de primaria," 2012.

Recibido: 6 de enero de 2019

Aceptado: 12 de julio de 2019

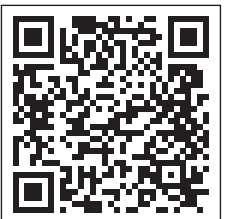

